\title{
Chromospheric activity of 9 Aurigae
}

\author{
Anthony B. Kaye ${ }^{1}$, Cristine Kristof ${ }^{1}$, and Richard O. Gray ${ }^{2}$ \\ ${ }^{1}$ Department of Physics, Texas Tech University \\ Lubbock, Texas 79409-1051, United States of America \\ email:anthony . kaye@ttu .edu \\ ${ }^{2}$ Department of Physics and Astronomy, Appalachian State University \\ Boone, North Carolina 28608, United States of America \\ email:grayro@appstate.edu
}

\begin{abstract}
The definition of $\gamma$ Dor stars as a new class of variable stars by Kaye et al. (1999) was based on a number of criteria, including the failure to detect any emission in the Ca II $\mathrm{H}$ \& K lines in these stars (Kaye \& Strassmeier 1998; KS98). Over the last 30 years, efforts continue to look for the blue edge of chromospheric activity. As a part of this effort, we put $\gamma$ Dor stars to the test to see if magnetic fields play a non-trivial role in their variability.
\end{abstract}

\section{Introduction}

The He I $D_{3}$ triplet has been known to be an indicator of chromospheric activity in main sequence stars as early as 1985 (Wolff et al. 1985); it is especially useful in stars where traditional signs of activity are absent (e.g., the Ca II H\&K lines in F stars; KS98). Excellent studies on this topic can be found in Wolff et al. $(1985,1986)$, Wolff \& Heasley (1987), Rachford (1997, 1998, 2000), and Rachford \& Foight (2009; hereafter RF09).

The $\gamma$ Dor stars were defined by Kaye et al. (1999) and their pulsation mechanism was identified by Guzik et al. (2000). To date, other than the null result of Kaye \& Strassmeier (1998), there has been no systematic effort to investigate chromospheric activity in these stars. RF09 measured the $D_{3}$ triplet of 9 Aur twice, but very few (if any) $\gamma$ Dor stars have enough $D_{3}$ measurements to carry out time series analysis to investigate variation in the $D_{3}$ line.

\section{Observations and methodology}

We obtained 196 high-resolution spectra of 9 Aur using the 0.9-meter Coudé Feed telescope and the Coudé spectrograph at the Kitt Peak National Observatory during 14 nights in December 1998. The IRAF-reduced spectra have a 2-pixel resolution of $0.21 \AA$, a 2-pixel signal-to-noise ratio (SNR) of $\sim 500$, and cover $315 \AA$ between 5840 and $6155 \AA$.

In addition, one classification-resolution spectrum of 9 Aur was acquired with the Gray/Miller Cassegrain spectrograph on the 0.8-m telescope at the Dark Sky Observatory at Appalachian State University. This spectrum has a 2-pixel resolution of $1.8 \AA$, an SNR $\sim 300$, and spans a range of $800 \AA$ centered on $4200 \AA$.

Measuring the equivalent width $\left(W_{\lambda}\right)$ of the $D_{3}$ triplet is complicated since it is contaminated by both photospheric and telluric lines. Therefore, we followed this procedure:

(a) We used an ATLAS12 model (Kurucz 1993) with the standard known physical parameters for the Sun to adjust the $g f$ values in the region of the $D_{3}$ triplet; the best model had the best fit with an observed spectrum of the Sun (Delbouille et al. 1990).

(b) We used the MD SIMPLEX method of Gray et al. (2001), ATLAS9/ATLAS12 models, and SPECTRUM (Gray \& Corbally 1994) to model the 9 Aur photosphere 
and determine $\left.T_{\text {eff }}, \log g\right)$, and $[M / H] ; \xi_{t}$ was fixed at $2.0 \mathrm{~km} \mathrm{~s}^{-1}$. We compared the model fluxes with published fluxes from the $T D-1$ satellite (Thompson et al. 1978), from Strömgren $u b y$ photometry, and from fluxes from the spectrophotometry of Breger (1976) to ensure the model represented the star well.

(c) This model photosphere was convolved with the measured projected radial velocity of $9 \operatorname{Aur}\left(17.0 \mathrm{~km} \mathrm{~s}^{-1}\right.$; Fekel 2003), a limb darkening coefficient of 0.6, and the two-pixel instrumental broadening. We then computed the $W_{\lambda}$ of the $D_{3}$ triplet area between $5874.826 \AA$ and $5876.781 \AA$. This allows us to subtract out all photospheric features in the area, leaving only the $D_{3}$ triplet and telluric lines.

(d) We then used a program that divides out the telluric lines with an "effective" air mass based upon the best telluric data of the observing run, smoothed with a 3-pixel low pass filter, rectified to a unit continuum, and measured each $W_{\lambda}$. Final $D_{3} W_{\lambda}$ 's were computed by simply subtracting this result from the result obtained in step (c).

\section{Conclusions}

Our average $W_{\lambda}$ is $14 \mathrm{~m} \AA$ and is consistent with the values reported by RF09. Using the $10 \mathrm{~m} \AA$ threshold criterion of Wolff et al. (1986), we conclude that 9 Aur is moderately chromospherically active. We observe statistically significant variations in the $D_{3}$ line, and propose that some variation in our measurements of the $D_{3}$ line may be due to variations caused by the ongoing g-mode pulsations or to previously undiscovered acoustic modulations. Using the models generated by Warner, Kaye, \& Guzik (2003), we are able to confirm the hypothesis of Kaye (1998) that claimed that at least some $\gamma$ Dor stars have Rossby numbers of order unity (for 9 Aur, $R o=2.47$ ), so magnetic fields likely play a more significant role than originally thought.

\section{References}

Breger, M. 1976, ApJS, 32, 7

Delbouille, L., Roland, G., \& Neven, L. 1990, Atlas photometrique du spectra solaire de $\lambda 3000$ $a \lambda 10000$ ' (Liège: Université de Liège, Institut d'Astrophysique)

Fekel, F. C. 2003, PASP, 115, 807

Gray, R. O. \& Corbally, C. J. 1994, AJ, 107, 742

Gray, R. O., Graham, P. W., \& Hoyt, S. R. 2001, AJ, 121, 2159

Guzik, J. A., Kaye, A. B., Bradley, P. A., Cox, A. N., \& Neuforge, C. 2000, ApJ, 542, L57

Kaye, A. B. 1998, Ph.D. thesis, Georgia State University

Kaye, A. B. \& Strassmeier, K. G. 1998, MNRAS, 294, L35

Kaye, A. B., Handler, G., Krisciunas, K., Poretti, E., \& Zerbi, F. M. 1999, PASP, 111, 840

Kurucz, R. L. 1993, CD-ROM 13, ATLAS9 Stellar Atmosphere Programs and $2 \mathrm{~km} / \mathrm{s}$ Grid (Cambridge: SAO)

Rachford, B. L. 1997, ApJ, 486, 994

Rachford, B. L. 1998, ApJ, 505, 255

Rachford, B. L. 2000, MNRAS, 315, 24

Rachford, B. L. \& Foight, D. R. 2009, ApJ, 698, 786

Thompson, G. I., Nandy, K., Jamar, C., et al. 1978, Catalog of Stellar Ultraviolet Fluxes (NSSDC/ADC Cat. 2059B) (London: Sci. Res. Council)

Warner, P. B., Kaye, A. B., \& Guzik, J. A. 2003, ApJ, 593, 1049

Wolff, S. C. \& Heasley, J. N. 1987, PASP, 99, 957

Wolff, S. C., Heasley, J. N., \& Varsik, J. 1985, PASP, 97, 707

Wolff, S. C., Boesgaard, A. M., \& Simon, T. 1986, ApJ, 310, 360 